\title{
Compensación de potencia reactiva en sistemas desbalanceados utilizando algoritmos genéticos
}

\author{
Reactive power compensation in unbalanced systems using genetic algorithms
}

\author{
Davel Borges Vasconcellos ${ }^{1} \quad$ Ignacio Pérez Abril ${ }^{2} \quad$ Vicente León Martínez $^{3}$
}

Recibido 3 de junio de 2011, aceptado 30 de julio de 2012

Received: June 3, $2011 \quad$ Accepted: July 30, 2012

\begin{abstract}
RESUMEN
La eficiencia energética de un sistema eléctrico depende en gran medida del factor de potencia con que opera y de una eficaz compensación de potencia reactiva si esta es necesaria. Una de las vías más utilizadas para efectuar la compensación del reactivo es el empleo de bancos de capacitores. La óptima aplicación de estos presupone tres aspectos: la selección de la capacidad más adecuada de los bancos, el tipo de compensador a utilizar, fijo o variable, así como la ubicación en el sistema de suministro eléctrico. En determinadas publicaciones, estos aspectos se tratan de forma independiente. Sin embargo, desde el punto de vista técnico ellos están mutuamente vinculados y todos poseen una implicación económica, lo que obliga a la necesidad de considerarlos en conjunto para un problema de optimización dado. Existen referencias de otros trabajos que desarrollan métodos que consideran estos aspectos de manera conjunta, pero presentan limitantes para ser aplicados en los sistemas de suministro eléctrico del sector terciario y donde predominan condiciones de desbalance. En este trabajo se presenta un método con el empleo de los algoritmos genéticos que posibilita una formulación más exacta del problema arrojando soluciones de mayor calidad. El programa desarrollado se soporta sobre software MATLAB, versión 7.5 (R2007b), utilizando las estructuras de programación de Genetic Algorithm and Direct Search Toolbox. La efectividad de su aplicación se muestra en casos de estudio correspondientes a sistemas de suministro eléctrico de dos hoteles pertenecientes al sector terciario en la provincia de Camagüey, Cuba.
\end{abstract}

Palabras clave: Compensación reactiva, modelos de compensación, bancos de capacitores, algoritmos genéticos, optimización de potencia reactiva, sistemas de suministro eléctrico.

\begin{abstract}
The energy efficiency of an electric system depends in great extent of the reactive power compensation that is carried out. One of the ways most used for reactive is power of capacitor banks.

A good selection of these, presupposes three aspects: the selection of the banks capacity, the compensator type to be used and the allocation in the power system.

These aspects are treated through independent methods. However, each one depends on the rest and all have an economic implication, which peads to the necessity of their consideration in the optimization problem itself. There are references of methods that consider the three aspects in a same problem, but these present restrictions in their applications for secondary power systems.

In the paper, we have developed a method with the use of genetic algorithms, which facilitates a more exact problem formulation. The method has been programmed in software MATLAB, version 7.5 (R2007b), using programming structures of Genetic Algorithm and Direct Search Toolbox.
\end{abstract}

1 Departamento de Ingeniería Eléctrica. Universidad de Camagüey. Circunvalación Norte Km 51⁄2. Camagüey, Cuba. E-mail: davel.borges@ reduc.edu.cu

2 Centro de Estudios Electroenergéticos. Universidad Central Marta Abreu de Las Villas. Carretera de Camajuaní Km 5¹⁄2. Santa Clara, Cuba. E-mail: iperez@uclv.edu.cu

3 Departamento de Ingeniería Eléctrica. Universidad Politécnica de Valencia. Camino de Vera s/n. Valencia, España.

E-mail: vleon@die.upv.es 
The effectiveness is shown in two zstudy cases of systems hotels, belonging to the commercial building consumer sector.

Keywords: Reactive compensation, compensation models, capacitor banks, genetic algorithms, reactive power optimizations, secondary power system.

\section{INTRODUCCIÓN}

La compensación de potencia reactiva es un fenómeno indispensable para la mejora de la eficiencia energética de los sistemas eléctricos [1]. Esta se realiza usualmente en dos subsistemas bien definidos: el subsistema de distribución primaria, perteneciente al proveedor eléctrico, a través del cual se hace llegar la energía disponible a los clientes, y el subsistema de suministro eléctrico o también denominado de distribución secundaria (de baja tensión por lo general), propiedad en muchos casos del cliente, siendo el eslabón final donde se satisfacen las necesidades energéticas de los receptores.

Son varias las ventajas que proporciona la compensación de potencia reactiva para el sistema eléctrico, pero en el caso del subsistema de distribución primaria se ve más asociado a la necesidad de reducir pérdidas de energía durante el transporte y mejorar el perfil de la tensión. Por su parte, en los sistemas de suministro eléctrico dicha compensación está relacionada con la mejora del factor de potencia, establecido en la ley de comercialización del servicio eléctrico del país, al ser caracterizados dichos sistemas por operar como entidades económicas independientes, que se relacionan contractualmente con las empresas de transmisión y distribución de energía eléctrica [2].

En los sistemas de suministro eléctrico la compensación de potencia reactiva se realiza usualmente mediante bancos de capacitores trifásicos balanceados fijos o variables (controlados) [1].

Los métodos de selección de tales dispositivos han sido basados en técnicas de optimización, clasificadas en: analíticas, de programación matemática, heurísticas y de inteligencia artificial, encontrándose dentro de estas últimas los algoritmos genéticos [3].

Las técnicas tradicionales (analíticas y de programación matemática) son determinísticas y requieren de una formulación matemática exhaustiva del modelo de compensación, a los efectos del empleo de la herramienta específica de optimización. Por ejemplo, si se pretende utilizar la técnica simplex, es necesario que el modelo de optimización esté representado por una función objetivo lineal, con restricciones de dominio lineal; en tanto si se requiere utilizar la técnica de programación cuadrática secuencial, la función objetivo deberá representarse a través de una ecuación cuadrática [2]. Esto, sin duda, es una importante limitante para la aplicación de estas técnicas, al exigir la adaptación del problema al modelo de solución. Un grupo importante de técnicas heurísticas también poseen este tipo de limitante [4].

Muchos problemas de la vida práctica muestran comportamientos complejos y las técnicas tradicionales de optimización de la matemática, incluso algunas heurísticas, no satisfacen la solución de los mismos, siendo recomendable el empleo de técnicas basadas en la inteligencia artificial [4].

Algunas de las condiciones de los fenómenos que muestran este comportamiento complejo son [4]:

> Capacidad de realizar transiciones entre diferentes formas de comportamiento.

$>$ Son sistemas en estado de no equilibrio.

$>$ Son sistemas abiertos.

$>$ Son sistemas no lineales y retroalimentados.

$>$ Posibilidad de pérdida de estabilidad.

$>$ Capacidad de autoorganización.

Tal es el caso del problema de compensación de la potencia reactiva en sistemas de suministro eléctrico.

En este sentido, se propone un método de compensación de potencia reactiva para sistemas de suministro eléctrico, partiendo de un conjunto de datos necesarios y de la consideración del tipo de compensación deseada, para luego dar paso al establecimiento del modelo matemático, el cual se resuelve con ayuda de un algoritmo genético simple implementado en el software MATLAB utilizando las estructuras de programación de Genetic Algorithm and Direct Search Toolbox. 
Para ello, se parte de un análisis de los métodos de optimización empleados en la compensación de potencia reactiva, para con posterioridad establecer el planteamiento del problema, así como su modelación matemática y solución a partir de un algoritmo genético, que constituye el núcleo central del método propuesto.

\section{ANÁLISIS TEÓRICO}

\section{Métodos de optimización de potencia reactiva}

A lo largo de los años se han empleado disímiles métodos de selección y ubicación de bancos de capacitores, basados en modelos de compensación, que han sido resueltos a partir del uso de diversas de las técnicas de optimización disponibles.

En la última década se pueden citar ocho de estos métodos relacionados en la bibliografía [2, 5-11].

La tendencia del estudio de los mismos los caracteriza teniendo en cuenta las siguientes condiciones iniciales:

$>$ Han sido utilizados para la compensación reactiva en sistemas de distribución primaria en su mayoría.

$>$ Se aplican sobre la consideración de modelos de cargas balanceadas.

$>$ Los registros de mediciones de los gráficos de carga son aproximados y se consideran constantes en los años de explotación (comportamiento patrón de la carga).

$>$ Los bancos de capacitores resultantes son balanceados en la mayoría de los casos.

$>$ El modelo de función de optimización no considera todos los efectos de la compensación de forma exacta, dado fundamentalmente porque:

- Separan los ahorros por concepto de factor de potencia de los ahorros por reducción de pérdidas de energía en el sistema.

- La estandarización de las unidades capacitoras es aproximada e incluso únicamente para múltiplos de una misma unidad de compensación.

- Presentan aproximaciones al considerar los costos de instalación y accesorios auxiliares.

En los bancos controlados solo se modela un control por tiempo, que en realidad no se utiliza normalmente en la práctica.

Los modelos de cargas que se han utilizado son aproximados.
Estos aspectos han sido el resultado de las limitaciones impuestas por las técnicas de optimización empleadas para la solución del problema de optimización.

\section{El problema de la compensación a partir de algoritmos genéticos}

Los algoritmos genéticos (AG) fueron introducidos en los años sesenta y actualmente forman parte de la familia de procedimientos de optimización basados en la inteligencia artificial [12].

Es una técnica de búsqueda basada en la teoría de la evolución de Darwin, que funciona análogo al proceso de cruzamiento biológico de cromosomas que comparten información genética para crear un nuevo individuo. Al final, llegan a obtener un individuo de alta adaptación, lo que en el argot matemático no significa una solución óptima, pero sí la "mejor solución" [12].

Se diferencian de los algoritmos de optimización tradicionales en cuanto a que:

$>$ Son algoritmos estocásticos. Dos ejecuciones distintas pueden dar dos soluciones distintas.

$>$ Son algoritmos de búsqueda múltiple, de los cuales es posible obtener varias soluciones.

$>$ La convergencia es poco sensible a la población inicial. No existen restricciones en el espacio de búsqueda.

> Son algoritmos intrínsecamente paralelos, capaces de operar simultáneamente con varias soluciones, lo que asegura por lo general un funcionamiento más rápido en las corridas.

$>$ Resultan menos afectados por los máximos locales (falsas soluciones).

Los algoritmos genéticos se han empleado de forma satisfactoria en diferentes ciencias en la solución de problemas de optimización complejos de espacio de búsqueda discretos, por lo que resultan ser una eficaz herramienta para la solución del problema de compensación de potencia reactiva en los sistemas eléctricos.

\section{Formulación del problema de adaptabilidad}

El problema en cuestión, a consideración de los autores, puede ser redactado de la manera siguiente:

Determinar la cantidad de unidades de compensación de cada capacidad disponible, necesarias para compensar de forma fija simétrica y asimétrica o escalonada simétrica la potencia reactiva en el 
sistema de suministro eléctrico, tal que se obtenga el máximo de Valor Actual Neto (VAN).

Como consideraciones del problema aproximado (condiciones iniciales) fueron planteadas las siguientes:

Serán considerados en el análisis todos los efectos de la compensación de reactivo, que puedan ser expresados desde el punto de vista económico (inversiones en los bancos, gastos de explotación, beneficios por la mejora del factor de potencia y pérdidas de energía, así como por la liberación de carga en caso necesario).

$>$ Los análisis de flujo de cargas serán considerados de forma trifásica, teniendo en cuenta la existencia de desbalances en el sistema.

$>$ El modelo de gráficos de cargas deberá permitir la consideración de más de un comportamiento patrón, en los períodos de tiempo necesarios para su caracterización más exacta.

> Para la compensación se utilizarán bancos de capacitores fijos (simétricos y asimétricos) o bancos controlados escalonados simétricos.

$>$ La compensación fija se aplicará en todos los posibles nodos del sistema, siempre de forma exclusiva o derivados de aquellos nodos con compensación escalonada simétrica.

$>$ La compensación escalonada simétrica no deberá utilizarse en nodos derivados con igual tipo de compensación.

$>$ Los efectos sobre la tensión del sistema (baja o alta) deberán ser considerados en el análisis, de modo tal que no se excedan los límites de desviaciones establecidos en las normas.

\section{Modelo propuesto}

Dados:

$>$ Datos del sistema eléctrico.

$>$ Datos del comportamiento de las cargas.

$>$ Datos de facturación del servicio eléctrico en el último año de explotación de la instalación.

$>$ Datos de los bancos de capacitores disponibles.

$>$ Datos de análisis económico.

Se necesita minimizar la función de adaptabilidad Fitness:

$$
\begin{aligned}
& \text { Fitness }=K t-(B f p+B c-C p-C a) \sum_{j=1}^{n} \frac{1}{(1+T D)^{j}}+\ldots \\
& \ldots+10^{7} \cdot \text { Penal }
\end{aligned}
$$

Donde:

$K t$ : Costos de inversión de los bancos de capacitores (se incluyen los costos asociados a las unidades de compensación en relación con el nivel de tensión, los costos auxiliares en armarios y accesorios, costos de los dispositivos de control y costos de montaje).

$B f p$ : Beneficios por la mejora del factor de potencia que incluye las pérdidas de energía eléctrica.

Cp: Gastos de explotación (pérdidas de los bancos).

Ca: Gastos de explotación (amortización de los bancos).

$T D$ : Tasa de descuento.

$n$ : Años de explotación.

Penal: Función de penalización.

La función de penalización incorpora las restricciones al espacio de búsqueda del algoritmo y es la suma de magnitudes constantes determinadas a partir de los aspectos siguientes:

$>$ Disponibilidad de unidades capacitoras.

$>$ Capacidad máxima de compensación (limitación del espacio de búsqueda).

$>$ Cumplimiento de las normativas de desviaciones de tensión (máxima y mínima).

Como se puede notar, la expresión (1) sin incluir las penalizaciones representa el Valor Actual Neto (invertido en signo) obtenido a partir de los efectos económicos de la compensación.

\section{Programación del procedimiento de solución} Con ayuda del MATLAB, versión 7.8 (R2009a), se programó el modelo de compensación, utilizando las estructuras de programación de Genetic Algorithm and Direct Search Toolbox.

El pseudocódigo específico del algoritmo diseñado para implementar el modelo de compensación es el siguiente:

1. [Datos] Lectura de datos necesarios.

2. [Inicio] Generar una población aleatoria de $n$ individuos (PopulationSize, $n$ ) con cromosomas, expresados en el vector fila $[x]$, codificados en 
forma binaria (PopulationType, 'bitstring') de longitud nvars de acuerdo al problema.

3. [Fitness] Evaluar la función Fitness (-VAN+Penal) para cada cromosoma $x$ de la población. En este caso, la calidad de la evaluación estará dada por la minimización de la misma.

4. [Nueva población] Crear una nueva población, repitiendo los siguientes pasos hasta que se cumpla la condición de parada, en este caso por el número de $m$ generaciones preestablecidas en el algoritmo (Generations, $m$ ).

a) [Selección] Seleccionar dos cromosomas padres de una población de acuerdo a su Fitness.

b) [Crossover] Con un porcentaje de cruzamiento (CrossoverFraction) crear una nueva descendencia.

c) [Mutación] Con un porcentaje de mutación de acuerdo con la función seleccionada (MutationFcns) modificar la nueva población.

d) [Aceptar] Colocar los nuevos descendientes en la nueva población.

5. [Remplazar] Usar la nueva población generada para una futura corrida del algoritmo.

6. [Test] Si la condición de salida se satisface, parar y retornar la mejor solución (cromosoma $x$ ) para la población actual.

7. [Loop] Regresar al paso 3.

Método de compensación de la potencia reactiva A partir de la formulación del problema de optimización y el modelo matemático obtenido se puede establecer el método de compensación, que se compone de los aspectos siguientes:

1. Obtención de los datos necesarios para la aplicación. Estos datos pueden ser obtenidos a partir de los registros documentales de las empresas o trabajos previos de obtención de los mismos.

2. Establecimiento del modelo de optimización deseado para determinar la cantidad de unidades de cada tipo necesarias para compensar bajo las condiciones de compensación previamente especificadas: a) Con bancos fijos simétricos.

b) Con bancos fijos simétricos y asimétricos.

c) Con bancos controlados simétricos.

d) Con bancos fijos simétricos de conjunto con bancos controlados simétricos.

e) Con bancos fijos simétricos y asimétricos de conjunto con bancos controlados simétricos.

3. Ajuste de los parámetros de corrida del algoritmo genético de optimización, para asegurar la convergencia. En este caso, se sugiere iniciar con los parámetros especificados a continuación y bajo el criterio de corridas sucesivas del algoritmo, comprobar la convergencia de la solución ante la variación de los parámetros. Los principales parámetros de corrida obtenidos en la experimentación para la solución del problema de la compensación a partir del algoritmo genético simple son:

$>$ Tamaño de la población: 100 individuos.

$>$ Tipo de variables de exploración: cadena de bit.

$>$ Criterio de parada: Por máximo número de generaciones (50) excedida.

$>$ Probabilidad de cruzamiento: 0.8

$>$ Función de mutación: tipo Gauss.

4. A partir de la cantidad de unidades de compensación de cada tipo y bajo las condiciones de compensación especificadas, seleccionar los parámetros técnicos de los dispositivos para cada variante de alternativa de solución (al menos 10) que maximice la función objetivo establecida en el modelo con una desviación máxima de un 5\%. Fundamentar en cada caso la factibilidad a partir del análisis económico, empleando como sugerencia los métodos del Valor Actual Neto (VAN) y el Plazo de Retorno de la Inversión $(P R I)$, así como los indicadores económicos que lo avalan (costos de inversión, gastos de explotación, beneficios por la mejora del factor de potencia y pérdidas, entre otros).

5. Efectuar la toma de decisiones por parte del especialista acerca de qué variante asumir, tomando en consideración factores principalmente relacionados con la disponibilidad real del proveedor de bancos de capacitores y la inversión inicial a realizar, establecida en la futura relación contractual.

6. Proceder a la adquisición y montaje de la variante de bancos de capacitores asumida. 


\section{Fase experimental}

Para validar el método de compensación, se realizaron dos tipos de ensayos:

I. Pruebas a escala del modelo y sus valores extremos de solución. La finalidad de estas pruebas es comprobar el funcionamiento correcto del algoritmo bajo condiciones iniciales de frontera, donde son evidentes o han sido estudiadas y se conocen las respuestas probables de solución.

II. Pruebas de comprobación en relación con otros modelos. En este caso, se comprueban los resultados del algoritmo, en relación con determinadas condiciones iniciales asumidas por otros modelos y tomadas en consideración desde el propio algoritmo propuesto, a fin de comprobar la superioridad de este.

En ambos ensayos se utilizaron dos instalaciones de suministro eléctrico reales, pertenecientes a hoteles del sector consumidor terciario de la República de Cuba, cuyos esquemas topológicos se muestran en las Figuras 1 y 2.

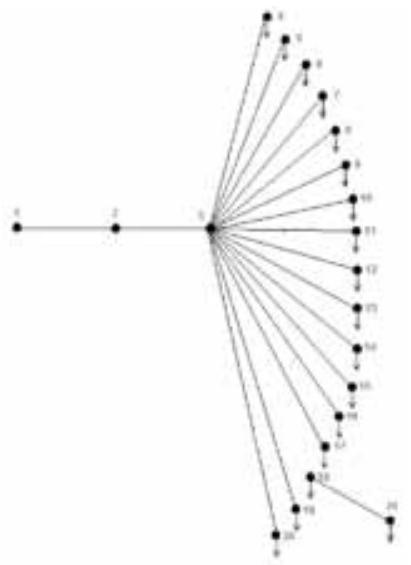

Figura 1. Esquema topológico del sistema eléctrico (caso de estudio 1).

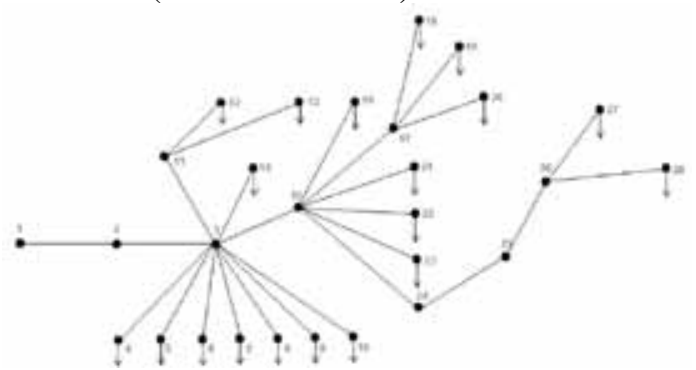

Figura 2. Esquema topológico del sistema eléctrico (caso de estudio 2).
Para las pruebas a escala del modelo y sus valores extremos de solución se implementaron 12 casos experimentales a razón de comprobar su efectividad, ante variaciones de las condiciones iniciales. Para asegurar una respuesta correcta (adecuada convergencia), se realizaron al menos 10 corridas de cada uno de los 12 casos.

Los casos experimentales de pruebas a escala con valores extremos del modelo y sus resultados fueron:

1. Considerando cero los gastos de explotación y los beneficios (solo se consideran los costos de inversión): Como es de resultar, no se ubica compensación en ninguno de los nodos del sistema.

2. Considerando cero los costos de inversión y por ende amortización: La compensación se ubica indistintamente en los nodos de mayores pérdidas de energía, asegurando un factor de potencia entre 0,96 y 1 .

3. Considerando cero los gastos de explotación (amortización y pérdidas de las unidades): La compensación se ubica indistintamente en los nodos de mayores pérdidas de energía, asegurando un factor de potencia entre $0,96 \mathrm{y}$ 1 , pero como regla ligeramente más bajo que en el caso anterior.

4. Considerando cero los gastos de pérdidas de las unidades: La compensación se ubica indistintamente en los nodos de mayores pérdidas de energía, con un resultado similar al caso anterior.

5. Considerando cero los gastos de amortización de las unidades: La compensación se ubica indistintamente los nodos de mayores pérdidas de energía, con un resultado similar al caso anterior.

6. Considerando cero los gastos de inversión y explotación: La compensación se ubica prácticamente en todos los nodos, asegurando un factor de potencia muy cercano a 1 .

7. Considerando solo los beneficios por la mejora del factor de potencia (sin pérdidas): La compensación se ubica a nivel global, asegurando un factor de potencia indistintamente entre 0,96 y 1. 
8. Considerando solo los beneficios por la reducción de pérdidas: La compensación se ubica prácticamente en todos los nodos, asegurando un factor de potencia muy cercano a 1 .

9. Considerando cero los gastos de explotación (amortización y pérdidas de las unidades), así como la reducción de pérdidas: La compensación se ubica a nivel global, asegurando un factor de potencia muy cercano a 0,96 .

10. Considerando cero los beneficios por la reducción de pérdidas: La compensación se ubica a nivel global, asegurando un factor de potencia cercano a 0,96 , similar al caso anterior.

11. Considerando cero los beneficios por la mejora del factor de potencia: No se ubica compensación en ninguno de los nodos del sistema.

12. Considerando cero los beneficios por la mejora del factor de potencia y los gastos de explotación: No se ubica compensación en ninguno de los nodos del sistema.

En la experimentación solo se utilizaron unidades compensadoras trifásicas, para lograr un adecuado nivel de comparación en relación con los modelos existentes.

Este algoritmo tal y como lo establece la literatura muestra un comportamiento estocástico. Las soluciones en diferentes corridas son por naturaleza diferentes, aunque convergen a un valor estable de la función objetivo $V A N$ con un porcentaje muy bajo de desviación (inferior al 5\%).

Los resultados obtenidos en las pruebas experimentales a escala con el modelo para valores extremos coinciden con los encontrados en la bibliografía sobre el tema, lo que corrobora la validez del modelo y el algoritmo implementado.

Para las pruebas de comprobación en relación con otros modelos se simularon las condiciones iniciales asumidas por los mismos y se compararon los resultados con respecto al modelo propuesto.

Los parámetros de calidad empleados en la comparación fueron el valor de la función de adaptabilidad (VAN), el tiempo de corrida del algoritmo y los resultados de convergencia.
Este ensayo de validación demostró que el modelo propuesto presenta:

$>$ Mejores resultados de la función objetivo (mayor o correcto $V A N$ ).

$>$ Menor tiempo de corrida promedio que los algoritmos tradicionales (menos cantidad de exploraciones en la búsqueda de la "mejor solución").

$>$ Absoluta convergencia con diferentes alternativas de solución (funcionamiento estocástico).

En el anexo aparecen los resultados de las dos aplicaciones.

Los resultados para la ubicación de bancos fijos (simétricos y/o asimétricos) aparecen en la Tabla 1 , donde solo se señalan los nodos donde se ubica la compensación para alguna de las diez corridas efectuadas. Las tres últimas filas de cada caso de estudio corresponden respectivamente a la potencia de compensación fija total, el Valor Actual Neto $(V A N)$ y el factor de potencia medio pesado global luego de la compensación.

En este caso, existe un predominio de compensación con bancos asimétricos, dado el desbalance de los circuitos del sistema. Se pudo comprobar que la compensación se ubica en aquellos nodos de mayores niveles de pérdidas de energía y en correspondencia con el grado de asimetría de la potencia reactiva.

Por su parte, en la Tabla 2 aparecen los resultados de cinco corridas para la ubicación global de un banco controlado, a partir de valores estándares de unidades capacitoras, que aseguran un factor de potencia luego de la compensación de máxima bonificación $(0,96)$, según la reglamentación vigente en la República de Cuba.

Para ambos casos se observa que se obtienen diferentes respuestas por corridas con un máximo y aproximado $V A N$, lo que refuerza el comportamiento estocástico del algoritmo, a la vez que posibilita al especialista la toma final de decisiones.

Los diferentes aspectos del modelo de compensación en el cual se basa el método de compensación propuesto han sido implementados de manera satisfactoria además en nueve servicios de asesoría brindada a empresas del territorio cubano. 


\section{CONCLUSIONES}

El método de compensación de potencia reactiva propuesto resulta más efectivo en la solución del problema de optimización que los métodos tradicionalmente empleados debido a que:

$>$ Posibilita una formulación más exacta de los efectos de la compensación.

$>$ No es necesaria la desestimación de efectos como los gastos de explotación de los bancos, que pueden modificar los resultados.

$>$ Asegura un espacio de búsqueda discreto, idóneo para este tipo de problema, dada la estandarización en la fabricación de las unidades de compensación.

$>$ Es posible de implementar con facilidad a partir de la Genetic Algorithm and Direct Search Toolbox del MATLAB presente en versiones 7.0 y superior.

El método de compensación de potencia reactiva para sistemas de suministro eléctrico de entidades del sector terciario, donde los desbalances pueden ser significativos, a partir de ubicar bancos fijos asimétricos, basado en la técnica de algoritmo genético, ofrece mejores resultados en relación con los métodos tradicionalmente empleados.

\section{AGRADECIMIENTOS}

Los autores agradecen a los profesores: Dr. Leonardo Casas Fernández de la Universidad Central de Las Villas, Cuba, y Dr. José Giner Gacía, de la Universidad de Valencia, España, por su ayuda en los basamentos del trabajo.

\section{REFERENCIAS}

[1] V. León Martínez, J. Montañana-Romeu, J. Roger Folch and A. Cazorla Navarro. "Why Reactive Compensators do not Improve the Efficiency Correctly in Unbalanced Circuits". XIX IMEKO World Congress Fundamental and Applied Metrology. Lisbon, Portugal. September 6-11, 2009.

[2] J. González Quintero. "Compensación de Potencia Reactiva en Sistemas Contaminados con Armónicos". Tesis para optar al grado de doctor. Universidad Central Marta Abreu de Las Villas. Santa Clara, Cuba. 2002.

[3] H.N. Ng, M.M.A. Salama and A.Y. Chikhani. "Classification of Capacitor Allocation Techniques". IEEE Transactions on Power Delivery. Vol. 15, Issue 1, pp. 387-392. January, 2000.

[4] G. Nicolis y I. Prigogine. "La estructura de lo complejo". Edición Castellana. Alianza Editorial. 1994 (Edición original, 1987).

[5] I. Pérez Abril and J. González Quintero. "VAR Compensation by Sequential Quadratic Programming". IEEE Transactions on Power Systems. Vol. 18, Issue 1, pp. 36-41. February, 2003.

[6] A. Faheem Zobaa and A. Aziz. "LC Compensators Based on Transmisión Loss Minimization for Nonlinear Loads”. IEEE Transactions on Power Delivery. Vol. 19, Issue 4, pp. 1740-1745. October, 2004.

[7] A. Faheem Zobaa. "Cost-Effective Applications of Power Factor Correction for Nonlinear Loads". IEEE Transactions on Power Delivery. Vol. 20, Issue 1, pp. 359365. January, 2005.

[8] P. Varilone, G. Carpinelli and A. Abur. "Capacitor Placement in Unbalanced Power Systems". 14 ${ }^{\text {th }}$ PSCC. Sevilla, España. June 24-28, 2002.

[9] M. Crispino, V. Di Vito, A. Russo and P. Varilone. "Decision Theory Criteria for Capacitor Placement in Unbalanced Distribution Systems". IEEE/PES Transmission and Distribution Conference and Exhibition. Asia and Pacific Dalian, China. 2005.

[10] M. Varadarajan and K.S. Swarup. "Differential Evolutionary Algorithm for Optimal Reactive Power Dispatch”. Electrical Power and Energy Systems 30, pp. 435-441. 2008.

[11] B. Milosevic and M. Begovié. "Capacitor Placement for Conservative Voltage Reduction on Distribution Feeders". IEEE Transactions on Power Delivery. Vol. 19, Issue 3, pp. 13601367. July, 2004.

[12] D.E. Goldberg. "Genetic Algorithms in Search, Optimization, and Machine Learning". Addison-Wesley Publishing Company, Inc. 1989. 


\section{ANEXO: RESULTADOS OBTENIDOS EN LAS APLICACIONES}

Tabla 1. Resultados por corridas con el empleo de bancos fijos (simétricos y/o asimétricos).

\begin{tabular}{|c|c|c|c|c|c|c|c|c|c|c|}
\hline \multicolumn{11}{|c|}{ Caso de estudio 1} \\
\hline \multirow{2}{*}{ Nodo } & \multicolumn{10}{|c|}{ Resultados por corridas. Potencia de compensación (CkVAr) } \\
\hline & 1 & 2 & 3 & 4 & 5 & 6 & 7 & 8 & 9 & 10 \\
\hline 3 & 70 & 85 & 75 & 55 & 55 & 50 & 95 & 70 & 75 & 85 \\
\hline 5 & 10 & 20 & & 15 & & & & & 5 & \\
\hline 6 & 20 & & 25 & $30^{*}$ & 20 & & 10 & 20 & 20 & \\
\hline 7 & & & & & & $55^{*}$ & & & & \\
\hline 19 & & & & \multirow[b]{2}{*}{100} & $25^{*}$ & & & 10 & & 20 \\
\hline Total & 100 & 105 & 100 & & 100 & 105 & 105 & 100 & 100 & 105 \\
\hline VAN (\$) & 64839 & 66736 & 66885 & 64805 & 64945 & 67037 & 66860 & 64918 & 65075 & 67119 \\
\hline FP desp. & 0.9604 & 0.9647 & 0.9603 & 0.9602 & 0.9604 & 0.9644 & 0.9644 & 0.9600 & 0.9602 & 0.9641 \\
\hline \multicolumn{11}{|c|}{ Caso de estudio 2} \\
\hline \multirow{2}{*}{ Nodo } & \multicolumn{10}{|c|}{ Resultados por corridas. Potencia de compensación (CkVAr) } \\
\hline & 1 & 2 & 3 & 4 & 5 & 6 & 7 & 8 & 9 & 10 \\
\hline 2 & & & 35 & 45 & 25 & 40 & 20 & 20 & 50 & 15 \\
\hline 3 & 45 & 55 & & & 20 & 5 & & & & 30 \\
\hline 15 & & & 10 & & & & 25 & $25^{*}$ & & \\
\hline Total & 100 & 105 & 100 & 100 & 100 & 105 & 105 & 100 & 100 & 105 \\
\hline VAN (\$) & 64839 & 66736 & 66885 & 64805 & 64945 & 67037 & 66860 & 64918 & 65075 & 67119 \\
\hline FP desp. & 0.9604 & 0.9647 & 0.9603 & 0.9602 & 0.9604 & 0.9644 & 0.9644 & 0.9600 & 0.9602 & 0.9641 \\
\hline
\end{tabular}

* Ubicación de compensación con bancos simétricos.

Tabla 2. Resultados por corridas con el empleo de bancos controlados simétricos.

\begin{tabular}{|c|c|c|c|c|c|c|c|c|c|c|}
\hline \multirow[t]{2}{*}{ Corrida } & \multirow{2}{*}{$\begin{array}{c}\text { Potencia de } \\
\text { compensación del } \\
\text { banco (CkVAr) }\end{array}$} & \multicolumn{7}{|c|}{$\begin{array}{c}\text { Cantidad de unidades por valores } \\
\text { estándares en CkVAr }\end{array}$} & \multirow[t]{2}{*}{ VAN (\$) } & \multirow[t]{2}{*}{ FP desp. } \\
\hline & & 150 & 120 & 90 & 60 & 45 & 30 & 15 & & \\
\hline \multicolumn{11}{|c|}{ Caso de estudio 1} \\
\hline 1 & 360 & & & 1 & 1 & 2 & 3 & 2 & 60391 & 0.96 \\
\hline 2 & 330 & & & 1 & 1 & 1 & 3 & 3 & 60634 & 0.96 \\
\hline 3 & 360 & & & 1 & 1 & 2 & 3 & 2 & 60408 & 0.96 \\
\hline 4 & 345 & & & 1 & 1 & 2 & 2 & 3 & 60525 & 0.96 \\
\hline 5 & 345 & & & 1 & 1 & 2 & 2 & 3 & 60524 & 0.96 \\
\hline \multicolumn{11}{|c|}{ Caso de estudio 2} \\
\hline 1 & 135 & & & & 1 & 1 & 0 & 2 & 69649 & 0.96 \\
\hline 2 & 165 & & & & 1 & 0 & 2 & 3 & 69190 & 0.96 \\
\hline 3 & 135 & & & & 1 & 1 & 0 & 2 & 69649 & 0.96 \\
\hline 4 & 135 & & & & 1 & 1 & 0 & 2 & 69649 & 0.96 \\
\hline 5 & 165 & & & & 1 & 1 & 1 & 2 & 69322 & 0.96 \\
\hline
\end{tabular}

